\title{
Surjective homomorphisms between surface braid groups
}

\author{
Lei Chen
}

April 29, 2019

\begin{abstract}
Let $P B_{n}\left(S_{g, p}\right)$ be the pure braid group of a genus $g>1$ surface with $p$ punctures. In this paper we prove that any surjective homomorphism $P B_{n}\left(S_{g, p}\right) \rightarrow P B_{m}\left(S_{g, p}\right)$ factors through one of the forgetful homomorphisms. We then compute the automorphism group of $P B_{m}\left(S_{g, p}\right)$, which gives a simpler proof of Irmak-Ivanov-McCarthy [IM03, Theorem 1]. Surprisingly, in contrast to the $n=1$ case, any automorphism of $P B_{n}\left(S_{g, p}\right)$ for $n>1$ is geometric.
\end{abstract}

\section{Introduction}

Given a surface $S$ and a positive number $n$, we denote the pure configuration space of $S$ by

$$
\operatorname{PConf}_{n}(S):=\left\{\left(x_{1}, \ldots, x_{n}\right) \in S^{n}: x_{i} \neq x_{j} \text { for } i \neq j\right\}
$$

There is a natural free action of the permutation group $\Sigma_{n}$ on $\operatorname{PConf}_{n}(S)$ given by permuting the coordinates and we refer to the corresponding quotient $\operatorname{Conf}_{n}(S):=\operatorname{PConf}_{n}(S) / \Sigma_{n}$ as the configuration space of $S$. Lastly, denote the $n$-strand pure braid group and the $n$-strand braid group of $S$ by

$$
P B_{n}(S):=\pi_{1}\left(\operatorname{PConf}_{n}(S)\right) \text { and } B_{n}(S):=\pi_{1}\left(\operatorname{Conf}_{n}(S)\right) .
$$

Our goal in this paper is to understand surjective homomorphisms between surface braid groups on different numbers of strands. For example, when $n \geq m>0$, there are natural maps $\operatorname{PConf}_{n}(S) \rightarrow \operatorname{PConf}_{m}(S)$ forgetting $n-m$ coordinates, which induce forgetful homomorphisms $P B_{n}(S) \rightarrow P B_{m}(S)$. In fact, up to automorphisms, these are the only surjective homomorphisms that arise:

Theorem 1.1 (surjective homomorphisms $P B_{n}(S) \rightarrow P B_{m}(S)$ ). Let $S$ be a (possibly noncompact) hyperbolic surface of finite type of genus at least 2. For $m, n>0$, every surjective homomorphism $F: P B_{n}(S) \rightarrow$ $P B_{m}(S)$ factors through some forgetful homomorphism, possibly post-composing with an automorphism of $P B_{m}(S)$.

In particular, when $m>n$, there is no surjective homomorphism $F: P B_{n}(S) \rightarrow P B_{m}(S)$. A group $G$ is called Hopfian if any epimorphism of $G$ is an isomorphism. Applying Theorem 1.1 in the case $n=m$ gives a proof of the fact that $P B_{n}(S)$ is Hopfian for $n>0$. Another way to show that $P B_{n}(S)$ is Hopfian is by showing that $P B_{n}(S)$ is a finitely generated residually finite group. This comes from an embedding of $P B_{n}(S)$ inside the automorphism group of a finite generated free group, which is residually finite by Baumslag [Bau63]. Another consequence of our theorem is the following: 
Corollary 1.2 (surjective homomorphisms $B_{n}(S) \rightarrow P B_{m}(S)$ ). Let $S$ be a (possibly noncompact) hyperbolic surface of finite type of genus at least 2 . For $m>0$ and $n>1$, there is no surjective homomorphism

$$
F: B_{n}(S) \rightarrow P B_{m}(S)
$$

Historically, the first nontrivial surjective homomorphism between braid groups arose from a classical miracle: "resolving the quartic". Indeed, let $R Q: \operatorname{Conf}_{4}(\mathbb{C}) \rightarrow \operatorname{Conf}_{3}(\mathbb{C})$ be the map given by

$$
R Q(a, b, c, d)=(a b+c d, a c+b d, a d+b c) .
$$

By computation, the induced homomorphism on fundamental groups $R Q_{*}: B_{4}(\mathbb{C}) \rightarrow B_{3}(\mathbb{C})$ is surjective. Theorem 1.1 says that there is no such miracle map between pure surface braid groups.

The readers may be wondering why we refer to $R Q_{*}$ as a miracle. One of the reasons behind this terminology is a result of Lin Lin04 saying that there is no surjective homomorphism $B_{n}(\mathbb{C}) \rightarrow B_{m}(\mathbb{C})$ when $n>m$ and $n>4$. To prove this, Lin classified all homomorphisms from $B_{n}(\mathbb{C})$ to $\Sigma_{m}$ when $n>m$ extending Artin's [Art47] classification of all homomorphisms from $B_{n}(\mathbb{C})$ to $\Sigma_{n}$. To get a similar result for surface braid groups, we would need to classify homomorphisms from $B_{n}(S)$ to $\Sigma_{m}$, extending Ivanov's Iva90, Theorem 1] classification of all homomorphisms from $B_{n}(S)$ to $\Sigma_{n}$. Based on Theorem 1.1, we have the following conjecture:

Conjecture 1.3. Let $S$ be a (possibly noncompact) hyperbolic surface of finite type of genus at least two. For $m, n>0$ and $m \neq n$, there is no surjective homomorphism

$$
F: B_{n}(S) \rightarrow B_{m}(S)
$$

In light of Theorem 1.1, in order to further understand all surjective homomorphisms between surface braid groups, we need to study the automorphism groups of surface braid groups $\operatorname{Aut}\left(P_{n}(S)\right)$. To this end, for $g>1$ let $\operatorname{Diff}^{ \pm}\left(S_{g, p, n}\right)$ be the group of diffeomorphisms of $S_{g}$ fixing two sets of punctures, one with $p$ points and the other with $n$ points including both orientation-preserving and orientation-reversing maps. Let $\operatorname{Mod}^{ \pm}\left(S_{g, p, n}\right):=\pi_{0}\left(\operatorname{Diff}^{ \pm}\left(S_{g, p, n}\right)\right)$ be the extended mapping class group of $S_{g, n, p}$. The following theorem computes $\operatorname{Aut}\left(P B_{n}\left(S_{g, p}\right)\right)$ except when $n=1$ and $p>0$.

Theorem 1.4 (The automorphism groups of $P B_{n}\left(S_{g, p}\right)$ and $B_{n}\left(S_{g, p}\right)$ ). Assume that $g>1$ and that either $n>1$ and $p>0$ or that $n=1$ and $p=0$. Then

$$
\operatorname{Mod}^{ \pm}\left(S_{g, p, n}\right) \cong \operatorname{Aut}\left(P B_{n}\left(S_{g, p}\right)\right) \cong \operatorname{Aut}\left(B_{n}\left(S_{g, p}\right)\right) .
$$

Remark. When $n=1$ and $p>0$, the statement of Theorem 1.4 is simply false. The group $P B_{1}\left(S_{g, p}\right)$ is a free group and there are many isomorphisms of $P B_{1}\left(S_{g, p}\right)$ that are not induced from diffeomorphisms. But as Theorem 1.4 shows, as long as $n>1$, every automorphism of $P B_{n}\left(S_{g, p}\right)$ is induced from a diffeomorphism of $S_{g, p}$.

It should be mentioned at this point that Theorem 1.4 has some predecessors: Irmak-Ivanov-McCarthy IIM03, Theorem 1] first computed the automorphism group of $P B_{n}\left(S_{g}\right)$ and showed that every element is geometric in the sense that it comes from a diffeomorphism of $S_{g}$. After this work was completed, we also found out that An An16] obtained Theorem 1.4 through a similar method as [IM03, Theorem 1]. Moreover, Kida-Yamagata [KY11] [KY13] showed that every injective homomorphism from a finite index subgroup of $P B_{n}\left(S_{g}\right)$ to itself is geometric. Nevertheless our method is new and appears to be much simpler than all 
of the above. In particular, we do not rely on Thurston's theory of surface homeomorphisms and canonical reduction systems. Instead, we use group cohomology and obstruction theory.

Acknowledgements The author is grateful to anonymous referees and Maxime Bergeron for suggestions on the writing. She would also like to extend her warmest thanks to Benson Farb for his extensive comments and for his invaluable support from start to finish.

\section{Proof of the classification of surjective homomorphism}

Let $S=S_{g, p}$ be a surface of genus $g$ with $p$ punctures and we denote the pure configuration space of $S$ by

$$
\operatorname{PConf}_{m}(S):=\left\{\left(x_{1}, \ldots, x_{m}\right) \in S^{m}: x_{i} \neq x_{j} \text { for } i \neq j\right\} .
$$

In this section, we will use the computations of $H^{*}\left(\operatorname{PConf}_{m}\left(S_{g, p}\right) ; \mathbb{Z}\right)$ to prove Theorem 1.1. Most computations here are similar to the computations in Chen [Che16, Lemma 3.4]. Consider the real codimensional two subspace $\triangle_{i j}$ of $S_{g, p}^{m}$, defined as

$$
\triangle_{i j}:=\left\{\left(x_{1}, \ldots, x_{m}\right) \in S_{g, p}^{m}: x_{i}=x_{j}\right\} .
$$

By Poincaré duality, the subspace $\triangle_{i j}$ determines a class $\left[\triangle_{i j}\right] \in H^{2}\left(S_{g, p}^{m} ; \mathbb{Z}\right)$.

Let $\left\{a_{k}^{\prime}, b_{k}^{\prime}\right\}_{k=1}^{g}$ be a symplectic basis for $H^{1}\left(S_{g} ; \mathbb{Z}\right)$ and $\left[S_{g}\right]^{\prime} \in H^{2}\left(S_{g, p} ; \mathbb{Z}\right)$ be the fundamental class. Fix a natural embedding $e: S_{g, p} \rightarrow S_{g}$. We define $a_{k}:=e^{*}\left(a_{k}^{\prime}\right)$ and $b_{k}:=e^{*}\left(b_{k}^{\prime}\right)$ for $k \in\{1, \ldots, g\}$. Let $\left[S_{g, p}\right]=e^{*}\left(\left[S_{g}\right]^{\prime}\right) \in H^{2}\left(S_{g, p} ; \mathbb{Q}\right)$ be the generator of the fundamental class; when $p>0$, we have that $\left[S_{g, p}\right]=0$. Let $p_{i}: S_{g, p}^{m} \rightarrow S_{g, p}$ be the projection onto the $i$ th coordinate.

Define $H_{i}:=p_{i}^{*}\left(H^{1}\left(S_{g, p} ; \mathbb{Z}\right)\right) \subset H^{1}\left(S_{g, p}^{m} ; \mathbb{Z}\right)$. There is a natural embedding $E: \operatorname{PConf}_{m}\left(S_{g, p}\right) \rightarrow S_{g, p}^{m}$. By the Künneth formula, we have

$$
H^{2}\left(S_{g, p}^{m} ; \mathbb{Z}\right) \cong \bigoplus_{i=1}^{n} \mathbb{Z} p_{i}^{*}\left[S_{g, p}\right] \oplus \bigoplus_{i \neq j} H_{i} \otimes H_{j}
$$

Denote the following composition of maps by $F$ :

$$
\bigoplus_{i=1}^{m} \mathbb{Z} p_{i}^{*}\left[S_{g, p}\right] \oplus \bigoplus_{i \neq j} H_{i} \otimes H_{j} \stackrel{\text { Künneth }}{\cong} H^{2}\left(S_{g, p}^{m} ; \mathbb{Z}\right) \stackrel{E^{*}}{\longrightarrow} H^{2}\left(\operatorname{PConf}_{m}\left(S_{g, p}\right) ; \mathbb{Z}\right)
$$

We have the following computations of $H^{1}\left(\operatorname{PConf}_{m}\left(S_{g, p}\right) ; \mathbb{Z}\right)$ and $H^{2}\left(\operatorname{PConf}_{m}\left(S_{g, p}\right) ; \mathbb{Z}\right)$.

Lemma 2.1. Let $g>1$ and $p, m>0$ be integers.

(1) We have the following isomorphisms:

$$
H^{1}\left(\operatorname{PConf}_{m}\left(S_{g, p}\right) ; \mathbb{Z}\right) \stackrel{E^{*}}{\cong} H^{1}\left(S_{g, p}^{m} ; \mathbb{Q}\right) \cong \bigoplus_{i=1}^{m} H_{i}
$$

(2) We have the following exact sequence

$$
0 \rightarrow \oplus_{1 \leq i<j \leq m} \mathbb{Z}\left[G_{i j}\right] \stackrel{d}{\rightarrow} \bigoplus_{i=1}^{n} \mathbb{Z} p_{i}^{*}\left[S_{g, p}\right] \oplus \bigoplus_{i \neq j} H_{i} \otimes H_{j} \stackrel{F}{\rightarrow} H^{2}\left(\operatorname{PConf}_{m}\left(S_{g, p}\right) ; \mathbb{Z}\right),
$$

where $d\left[G_{i j}\right]=p_{i}^{*}\left[S_{g, p}\right]+p_{j}^{*}\left[S_{g, p}\right]+\sum_{k=1}^{g}\left(p_{i}^{*} a_{k} \otimes p_{j}^{*} b_{k}-p_{i}^{*} b_{k} \otimes p_{j}^{*} a_{k}\right)$. 
Proof. By Totaro [Tot96, Theorem 1], there is a spectral sequence converging to $H^{*}\left(\operatorname{PConf}_{m}\left(S_{g, p}\right) ; \mathbb{Q}\right)$ whose $E_{2}$ term is a bigraded algebra

$$
H^{*}\left(S_{g, p}^{m} ; \mathbb{Z}\right)\left[G_{i j}\right],
$$

where $H^{r}\left(S_{g, p}^{m} ; \mathbb{Z}\right)$ has degree $(r, 0)$ and $G_{i j}$ are generators of degree $(0,1)$ for $1 \leq i, j \leq m$ and $i \neq j$, modulo the following relations:

- $G_{i j}=G_{j i},\left(G_{i j}\right)^{2}=0$,

- $G_{i j} G_{i k}+G_{j k} G_{j i}+G_{k i} G_{k j}=0$ for $i, j, k$ distinct,

- $p_{i}^{*}(x) G_{i j}=p_{j}^{*}(x) G_{i j}$ for $x \in H^{*}\left(S_{g, p} ; \mathbb{Z}\right)$.

The differential is given by $d_{2}\left(G_{i j}\right)=\left[\triangle_{i j}\right]$. The following graph is a part of this spectral sequence:

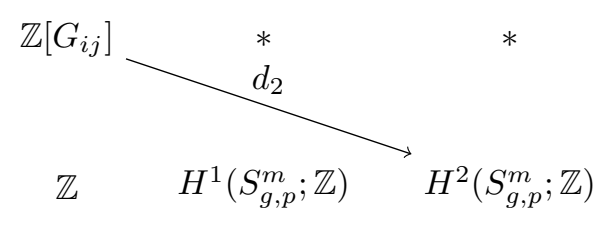

By Milnor-Stasheff [MS74, Section 11],

$\left[\triangle_{i j}\right]=p_{i}^{*}\left[S_{g, p}\right]+p_{j}^{*}\left[S_{g, p}\right]+\sum_{k=1}^{g}\left(p_{i}^{*} a_{k} \otimes p_{j}^{*} b_{k}-p_{i}^{*} b_{k} \otimes p_{j}^{*} a_{k}\right) \in \bigoplus_{i=1}^{n} \mathbb{Z} p_{i}^{*}\left[S_{g, p}\right] \oplus \bigoplus_{i \neq j} H_{i} \otimes H_{j} \stackrel{\text { Künneth }}{\cong} H^{2}\left(S_{g, p}^{m} ; \mathbb{Z}\right)$

When $g>1$, elements of the finite set

$$
\left\{\sum_{k=1}^{g}\left(p_{i}^{*} a_{k} \otimes p_{j}^{*} b_{k}-p_{i}^{*} b_{k} \otimes p_{j}^{*} a_{k}\right): 1 \leq i, j \leq m \text { and } i \neq j\right\}
$$

are linearly independent by the independence of direct sums. Thus $\left[\triangle_{i j}\right]$ are linearly independent; i.e. $d_{2}$ is injective. Then this lemma follows from the convergence of the above spectral sequence.

We have the following property about the cup product structure of $H^{*}\left(\operatorname{PConf}_{m}\left(S_{g, p}\right) ; \mathbb{Z}\right)$.

Lemma 2.2. For two independent elements $x, y \in H^{1}\left(\operatorname{PConf}_{m}\left(S_{g, p}\right) ; \mathbb{Z}\right)$, if $x \smile y=0$, then there exists $i \in\{1, \ldots, m\}$ such that either $x \in E^{*}\left(H_{i}\right)$ or $y \in E^{*}\left(H_{i}\right)$.

Proof. Since $E^{*}: \bigoplus_{i=1}^{m} H_{i} \rightarrow H^{1}\left(\operatorname{PConf}_{m}\left(S_{g, p}\right) ; \mathbb{Z}\right)$ is an isomorphism by Lemma2.1, we can find $x^{i}, y^{i} \in H_{i}$ for each $1 \leq i \leq m$ such that $x=E^{*}\left(x^{1}+\ldots+x^{m}\right)$ and $y=E^{*}\left(y^{1}+\ldots+y^{m}\right)$. The multiplication of $x$ and $y$ satisfies the following:

$x \smile y=F\left(x^{1} \smile y^{1}+\ldots+x^{n} \smile y^{n}+\sum_{i \neq j}\left(x^{i} \otimes y^{j}-y^{i} \otimes x^{j}\right)\right) \in \bigoplus_{i=1}^{n} \mathbb{Z} p_{i}^{*}\left[S_{g, p}\right] \oplus \bigoplus_{i \neq j} H_{i} \otimes H_{j} \stackrel{F}{\rightarrow} H^{2}\left(\operatorname{PConf}_{n}\left(S_{g, p}\right) ; \mathbb{Z}\right)$.

By $x \smile y=0 \in H^{2}\left(\operatorname{PConf}_{n}\left(S_{g, p}\right) ; \mathbb{Z}\right)$ and Lemma 2.1, there exists integers $\left\{k_{i, j}\right\}$ such that

$$
x^{1} \smile y^{1}+\ldots+x^{n} \smile y^{n}+\sum_{i \neq j}\left(x^{i} \otimes y^{j}-y^{i} \otimes x^{j}\right)=\sum k_{i, j} d\left[G_{i, j}\right] \in \bigoplus_{i=1}^{n} \mathbb{Z} p_{i}^{*}\left[S_{g, p}\right] \oplus \bigoplus_{i \neq j} H_{i} \otimes H_{j} .
$$

By the independence of all the terms in $\bigoplus_{i=1}^{n} \mathbb{Z} p_{i}^{*}\left[S_{g, p}\right] \oplus \bigoplus_{i \neq j} H_{i} \otimes H_{j}$, we have

$$
x^{i} \otimes y^{j}-y^{i} \otimes x^{j}=k_{i, j}\left(\sum_{k}\left(p_{i}^{*} a_{k} \otimes p_{j}^{*} b_{k}-p_{i}^{*} b_{k} \otimes p_{j}^{*} a_{k}\right)\right) \text { for all } i, j .
$$


Claim 2.3. We have that $k_{i, j}=0$ for all $i \neq j$.

Proof. We will prove this claim by contradiction. Assume that $k_{i, j} \neq 0$. In $H_{i}$, elements $p_{i}^{*} a_{1}, \ldots, p_{i}^{*} a_{g}$ span a subspace $A_{i}$ and $p_{i}^{*} b_{1}, \ldots, p_{i}^{*} b_{g}$ span a subspace $B_{i}$. Since $H_{i}$ is a $\mathbb{Z}$-free module, there is a projection $s_{i}: H_{i} \rightarrow A_{i} \oplus B_{i}$. Let $s_{i}\left(x^{i}\right)=x_{A}^{i}+x_{B}^{i} \in A_{i} \oplus B_{i}$ and $s_{i}\left(y^{i}\right)=y_{A}^{i}+y_{B}^{i} \in A_{i} \oplus B_{i}$. By equation (1D) and the projection by $s_{i} \otimes s_{j}$, we have that

$\left(x_{A}^{i}+x_{B}^{i}\right) \otimes\left(y_{A}^{j}+y_{B}^{j}\right)-\left(y_{A}^{i}+y_{B}^{i}\right) \otimes\left(x_{A}^{j}+x_{B}^{j}\right)=k_{i, j}\left(\sum_{k=1}^{g}\left(p_{i}^{*} a_{k} \otimes p_{j}^{*} b_{k}-p_{i}^{*} b_{k} \otimes p_{j}^{*} a_{k}\right)\right) \in\left(A_{i} \oplus B_{i}\right) \otimes\left(A_{j} \oplus B_{j}\right)$.

Since $\left(A_{i} \oplus B_{i}\right) \otimes\left(A_{j} \oplus B_{j}\right)=A_{i} \otimes A_{j} \oplus A_{i} \otimes B_{j} \oplus B_{i} \otimes A_{j} \oplus B_{i} \otimes B_{j}$, we have

- (a) $x_{A}^{i} \otimes y_{A}^{j}-y_{A}^{i} \otimes x_{A}^{j}=0$

- (b) $x_{B}^{i} \otimes y_{B}^{j}-y_{B}^{i} \otimes x_{B}^{j}=0$

- (c) $x_{A}^{i} \otimes y_{B}^{j}-y_{A}^{i} \otimes x_{B}^{j}=k_{i, j}\left(\sum_{k} p_{i}^{*} a_{k} \otimes p_{j}^{*} b_{k}\right)$

- (d) $x_{B}^{i} \otimes y_{A}^{j}-y_{B}^{i} \otimes x_{A}^{j}=k_{i, j}\left(\sum_{k} p_{i}^{*} b_{k} \otimes p_{j}^{*} a_{k}\right)$.

We claim that $k_{i, j}\left(\sum_{k} p_{i}^{*} a_{k} \otimes p_{j}^{*} b_{k}\right) \in A_{i} \otimes B_{j}$ is not a simple tensor of two elements. Assume the contrary that there exists $\lambda_{d}, \mu_{d}$ for all $d \in\{1, \ldots, m\}$ such that $z=\sum_{d} \lambda_{d} p_{i}^{*} a_{d}$ and $w=\sum_{d} \mu_{d} p_{i}^{*} b_{d}$ satisfies that $z \otimes w=k_{i, j}\left(\sum_{k} p_{i}^{*} a_{k} \otimes p_{j}^{*} b_{k}\right)$. Comparing the coefficient of $p_{i}^{*} a_{k} \otimes p_{j}^{*} b_{k}$, we know that $\lambda_{k} \neq 0$ and $\mu_{k} \neq 0$ for any $k$. Since $g>1$, the coefficient of $p_{i}^{*} a_{1} \otimes p_{j}^{*} b_{2}$ is nonzero, which is a contradiction.

By equation (c) and the fact that $\sum_{k} p_{i}^{*} a_{k} \otimes p_{j}^{*} b_{k} \in A_{i} \otimes B_{j}$ is not a simple tensor, we know that $x_{A}^{i}, y_{B}^{j}, y_{A}^{i}$ and $x_{B}^{j}$ are all nonzero. For the same reason, $x_{B}^{i}, y_{A}^{j}, y_{B}^{i}$ and $x_{A}^{j}$ are also all nonzero. Equation (a) says that there exists $\mu$ such that $x_{A}^{i}=\mu y_{A}^{i}$ and $\mu y_{A}^{j}=x_{A}^{j}$; equation (b) says that there exists $\mu^{\prime}$ such that $x_{B}^{i}=\mu^{\prime} y_{B}^{i}$ and $\mu^{\prime} y_{B}^{j}=x_{B}^{j}$. Therefore

$$
x_{A}^{i} \otimes y_{B}^{j}-y_{A}^{i} \otimes x_{B}^{j}=\mu y_{A}^{i} \otimes y_{B}^{j}-y_{A}^{i} \otimes \mu^{\prime} y_{B}^{j}=\left(\mu-\mu^{\prime}\right) y_{A}^{i} \otimes y_{B}^{j},
$$

which contradicts the fact that $\sum_{k} p_{i}^{*} a_{k} \otimes p_{j}^{*} b_{k} \in A_{i} \otimes B_{j}$ is not a simple tensor. Thus the claim holds.

Therefore $x^{i} \otimes y^{j}-y^{i} \otimes x^{j}=0 \in H_{i} \otimes H_{j}$. Assume that $x \notin H_{i}$ for any $i$; i.e. two coordinates of $x$ are nonzero. Without loss of generality, we assume that $x^{1} \neq 0$ and $x^{2} \neq 0$. We break the proof into the following cases.

- Case 1) $y^{1} \neq 0$ and $y^{1}$ is not proportional to $x^{1}$. Then $x^{1} \otimes y^{j}=y^{1} \otimes x^{j} \in H_{1} \otimes H_{j}$ implies that $y^{j}=0$ and $x^{j}=0$ for all $j \neq 1$, which contradicts to the assumption that $x_{2} \neq 0$.

- Case 2) $y^{1} \neq 0$ and $x^{1}=\mu y^{1}$ for $\mu \neq 0$. Then $x^{1} \otimes y^{j}=y^{1} \otimes x^{j} \in H_{1} \otimes H_{j}$ implies that $x^{j}=\mu y^{j}$ for all $j$. Therefore

$$
x=x^{1}+\ldots+x^{m}=\mu y^{1}+\ldots+\mu y^{m}=\mu y,
$$

which contradicts the fact that $x$ and $y$ are independent.

- Case 3) $y^{1}=0$. Then $x^{1} \otimes y^{j}=y^{1} \otimes x^{j} \in H_{1} \otimes H_{j}$ implies that $y^{j}=0$ for all $j$, which contradicts to the fact that $x$ and $y$ are independent.

We define $P B_{n}\left(S_{g, p}\right):=\pi_{1}\left(\operatorname{PConf}_{n}\left(S_{g, p}\right)\right)$ and let $P_{i}:=\left(p_{i} \circ E\right)_{*}: P B_{n}\left(S_{g, p}\right) \rightarrow \pi_{1}\left(S_{g, p}\right)$ be the induced map on the fundamental groups of the projection to the $i$ th coordinate. 
Proposition 2.4. For $g>0$ and $p, n \geq 0$, the space $\operatorname{PConf}_{n}\left(S_{g, p}\right)$ is a $K(\pi, 1)$-space and $\operatorname{Ker}\left(P_{i}\right)$ is finitely generated.

Proof. We will prove that $\operatorname{PConf}_{n}\left(S_{g, p}\right)$ is a $K(\pi, 1)$-space for any $p \geq 0$ by induction on $n$. For $n=1$, the space $\operatorname{PConf}_{n}\left(S_{g, p}\right)=S_{g, p}$ is a $K(\pi, 1)$-space for any $p$. Assume that $\operatorname{PConf}_{n-1}\left(S_{g, p}\right)$ is a $K(\pi, 1)$-space for any $p$ for $n>1$. The forgetful map $p_{i} \circ E$ gives the following fiber bundle

$$
\operatorname{PConf}_{n-1}\left(S_{g, p+1}\right) \rightarrow \operatorname{PConf}_{n}\left(S_{g, p}\right) \stackrel{p_{i} \circ E}{\longrightarrow} S_{g, p} .
$$

Since both $\mathrm{PConf}_{n-1}\left(S_{g, p+1}\right)$ and $S_{g, p}$ are $K(\pi, 1)$-spaces, we have that $\operatorname{PConf}_{n}\left(S_{g, p}\right)$ is a $K(\pi, 1)$-space. Then the induction axiom implies that $\operatorname{PConf}_{n}\left(S_{g, p}\right)$ is a $K(\pi, 1)$-space for any $n$. Fiber bundle (2) induces the following short exact sequence on fundamental groups:

$$
1 \rightarrow \mathrm{PB}_{n-1}\left(S_{g, p+1}\right) \rightarrow \mathrm{PB}_{n}\left(S_{g, p}\right) \stackrel{P_{i}}{\longrightarrow} \pi_{1}\left(S_{g, p}\right) \rightarrow 1 .
$$

By the short exact sequence (3), we obtain $\operatorname{Ker}\left(P_{i}\right)=P B_{n-1}\left(S_{g, p+1}\right)$. Using (3), it is straightforward to prove that $P B_{n}\left(S_{g, p}\right)$ is finitely generated by induction on $n$. Therefore $\operatorname{Ker}\left(P_{i}\right)$ is finitely generated.

Now, we proceed to the key lemma.

Lemma 2.5. For $g>1$ and $p, n \geq 0$, a homomorphism

$$
R: P B_{n}\left(S_{g, p}\right) \rightarrow \pi_{1}\left(S_{g, p}\right)
$$

either factors through $P_{i}$ for some $i \in\{1, \ldots, n\}$ or has cyclic image.

Proof. The proof of this lemma uses the same idea as F.E.A. Johnson Joh99]. The method can also be found in Salter Sal15, Lemma 3.3 and 3.4]. We use group cohomology in what follows. Since all spaces we consider are $K(\pi, 1)$-spaces, we freely use the computation of the cohomology of the spaces as the cohomology of the corresponding groups. By the classification of subgroups of $\pi_{1}\left(S_{g, p}\right)$, if $\operatorname{Image}(R) \nsucceq \mathbb{Z}$, then Image $(R)$ is either a free group $F_{k}$ with $k>1$ or a surface group $\pi_{1}\left(S_{h}\right)$ such that $h \geq g>1$. In both cases, there are independent elements $x, y \in H^{1}(\operatorname{Image}(R) ; \mathbb{Z})$ such that $x \smile y=0$. Denote by $S: P B_{n}\left(S_{g, p}\right) \rightarrow \operatorname{Image}(R)$ the map to the image of $R$, which is surjective by definition. Then $S^{*}(x), S^{*}(y) \in H^{1}\left(P B_{n}\left(S_{g, p}\right) ; \mathbb{Q}\right)$ are independent and $S^{*}(x) \smile S^{*}(y)=0$. By Lemma 2.2, we have either $S^{*}(x) \in P_{i}^{*}\left(H^{1}\left(\pi_{1}\left(S_{g, p}\right) ; \mathbb{Z}\right)\right)$ or $S^{*}(y) \in P_{i}^{*}\left(H^{1}\left(\pi_{1}\left(S_{g, p}\right) ; \mathbb{Z}\right)\right)$ for some $i$. Without loss of generality, assume that $S^{*}(x)=P_{i}^{*}\left(x^{\prime}\right)$. We have the following commutative diagram by the identification $H^{1}(\ldots ; \mathbb{Z}) \cong \operatorname{Hom}($ _- $\mathbb{Z})$,

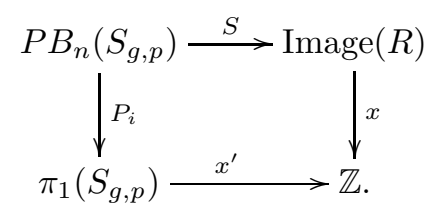

By Proposition 2.4 the group $\operatorname{Ker}\left(P_{i}\right)$ is a finitely generated normal subgroup of $P B_{n}\left(S_{g, p}\right)$. Since $S$ is surjective, the image $S\left(\operatorname{Ker}\left(P_{i}\right)\right)$ is also a finite generated normal subgroup of $\operatorname{Image}(R)$. However every finitely generated normal subgroup of $\operatorname{Image}(R)$ is either of finite index or trivial; see e.g. F.E.A. Johnson Joh99, Property (D6)]. If $S\left(\operatorname{Ker}\left(P_{i}\right)\right)<\operatorname{Image}(R)$ is of finite index, then after composing with $x$, the image $x \circ S\left(\operatorname{Ker}\left(P_{i}\right)\right)$ will be of finite index in $\mathbb{Z}$. This is a contradiction because $x \circ S\left(\operatorname{Ker}\left(P_{i}\right)\right)=x^{\prime} \circ P_{i}\left(\operatorname{Ker}\left(P_{i}\right)\right)=$ $\{1\}$. Therefore $S\left(\operatorname{Ker}\left(P_{i}\right)\right)=1$; i.e. $S$ factors through $P_{i}$. 
Now we are ready to prove Theorem 1.1 saying that for $m, n>0$ and $g>1$, every surjective homomorphism $F: P B_{n}\left(S_{g, p}\right) \rightarrow P B_{m}\left(S_{g, p}\right)$ factors through some forgetful homomorphism, possibly post-composing with an automorphism of $P B_{m}\left(S_{g, p}\right)$.

Proof of Theorem 1.1. We will prove Theorem 1.1] by induction on $m$. There are two things to be proved: every surjective homomorphism $F: P B_{n}\left(S_{g, p}\right) \rightarrow P B_{m}\left(S_{g, p}\right)$ factors through a forgetful homomorphism for any $m$ and $n$ and that $P B_{m}\left(S_{g}\right)$ is Hopfian for any $m$. The case $m=1$ is a result of Lemma 2.5 and the classical fact that $\pi_{1}(S)$ is Hopfian; see e.g. Hempel [Hem72]. We assume that when $m<k$, Theorem 1.1 is true. For $m=k$, let $f: P B_{n}\left(S_{g, p}\right) \rightarrow P B_{k}\left(S_{g, p}\right)$ be a surjective homomorphism. By postcomposing with a projection $p r: P B_{k}\left(S_{g, p}\right) \rightarrow P B_{k-1}\left(S_{g, p}\right)$, we obtain a new surjective homomorphism $p r \circ f: P B_{n}\left(S_{g, p}\right) \rightarrow P B_{k-1}\left(S_{g, p}\right)$. By the inductive hypothesis, $p r \circ f$ factors through some forgetful map, possibly post-composing with an automorphism of $P B_{k-1}\left(S_{g, p}\right)$. Therefore we have the following commutative diagram:

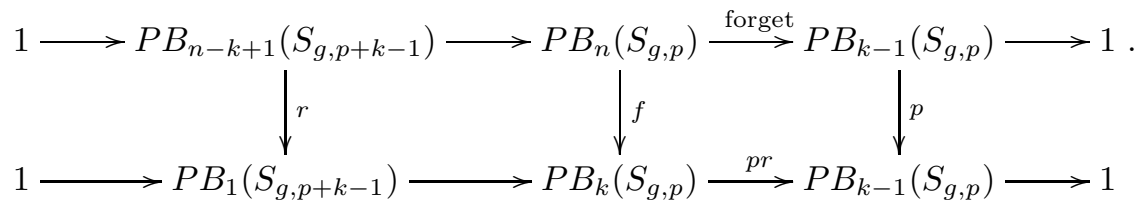

Since $P B_{n-k+1}\left(S_{g, p+k-1}\right) \rightarrow P B_{1}\left(S_{g, p+k-1}\right)$ factors through a forgetful homomorphism, which is the $m=1$ case of the theorem, we show that $f$ factors through a forgetful homomorphism. When $n=k$, both $r$ and $p$ are isomorphisms because of the inductive hypothesis. Therefore $f$ is an isomorphism by the five lemma. We finish the proof by the induction axiom.

Define $B_{n}(S):=\pi_{1}\left(\operatorname{PConf}_{n}(S) / \Sigma_{n}\right)$, where $\Sigma_{n}$ acts on $\operatorname{PConf}_{n}(S)$ by permuting coordinates. Now we are ready to prove Corollary 1.2 saying that there is no surjective homomorphism from $B_{n}\left(S_{g, p}\right)$ to $P B_{m}\left(S_{g, p}\right)$ when $g>1, n>1$ and $m>0$.

Proof of Corollary 1.2. Set $S=S_{g, p}$. We plan to prove that there is no surjective homomorphism $f$ : $B_{n}\left(S_{g, p}\right) \rightarrow P B_{m}\left(S_{g, p}\right)$ by contradiction. Assume the opposite that there is a surjective homomorphism $f: B_{n}\left(S_{g, p}\right) \rightarrow P B_{m}\left(S_{g, p}\right)$. By projecting to some coordinate, we obtain a surjective homomorphism $F: B_{n}(S) \rightarrow \pi_{1}(S)$. By post-composing with the embedding $p_{*}: P B_{n}(S) \rightarrow B_{n}(S)$, which is induced by the projection $p: \operatorname{PConf}_{n}(S) \rightarrow \operatorname{Conf}_{n}(S)$, we obtain a map $R=F \circ p_{*}: P B_{n}(S) \rightarrow \pi_{1}(S)$. Since $F$ is surjective, $\operatorname{Im}(R)$ is an index $n$ ! subgroup $H<\pi_{1}(S)$, where $H=\pi_{1}\left(S^{\prime}\right)$ is the fundamental group of an $n$ ! cover $S^{\prime}$ of $S$. We have the following inequalities of the first Betti numbers of $S$ and $S^{\prime}$ :

$$
\begin{aligned}
b_{1}\left(S^{\prime}\right) & \geq 1-\chi\left(S^{\prime}\right) & & b_{1}\left(S^{\prime}\right)=b_{0}\left(S^{\prime}\right)+b_{2}\left(S^{\prime}\right)-\chi\left(S^{\prime}\right) \geq 1-\chi\left(S^{\prime}\right) \\
& =1-n ! \chi(S) & & S^{\prime} \text { is } n ! \text {-cover of } S \\
& \geq 1-2 \chi(S) & & \chi(S) \text { is negative } \\
& \geq 3-\chi(S) & & g>1 \text { implies that } \chi(S) \leq-2 \\
& \geq 1+b_{1}(S) & & b_{1}(S)=b_{0}(S)+b_{2}(S)-\chi(S) \leq 2-\chi(S) .
\end{aligned}
$$

Since $R$ does not have a cyclic image, we know that $R=Q \circ P_{i}$ for some $i \in\{1, \ldots, n\}$ and some map $Q: \pi_{1}(S) \rightarrow \pi_{1}(S)$ by Lemma 2.5. Since $P_{i}$ is surjective, we know that $\operatorname{Im}(Q)=\operatorname{Im}(R)=H$. However $Q$ cannot be surjective onto the image $\pi_{1}\left(S^{\prime}\right)$ because $b_{1}(S)<b_{1}\left(S^{\prime}\right)$, which is a contradiction. 


\section{The automorphism group of $\mathrm{PB}_{n}\left(S_{g, p}\right)$}

In this section, we will compute the automorphism group of $\mathrm{PB}_{n}\left(S_{g, p}\right)$. The key point is to use the existence of pseudo-Anosov elements in the point-pushing subgroup. Before the proof of the result, we will introduce 3 classical results we will use in the proof. Firstly, we have the following result of Handel-Thurston [HT85, Lemma 2.2].

Theorem 3.1 (HT85]). A pseudo-Anosov element of the mapping class group does not fix any nonperipheral isotopy class of curves (including nonsimple curves).

Another ingredient is Kra's construction [Kra81]. Let $S$ be a finite type surface possibly with punctures. The extended mapping class group $\operatorname{Mod}^{ \pm}(S)$ is defined to be the group of isotopy classes of diffeomorphisms of $S$ fixing the punctures as a set. Later, we will define other types of extended mapping class groups by specifying exactly how they preserve the punctures. Let $b \in S$. Denote by $\operatorname{Mod}^{ \pm}(S, b)$ the extended mapping class group of $S$ fixing a point $b$; in particular $\operatorname{Mod}^{ \pm}(S, b)$ fixes the punctures of $S$ as a set and the point $b$. The following is the Birman exact sequence for $S$ (see e.g. Farb-Margalit [FM12, Section 4.2]):

$$
1 \rightarrow \pi_{1}(S, b) \stackrel{\text { Push }}{\longrightarrow} \operatorname{Mod}^{ \pm}(S, b) \rightarrow \operatorname{Mod}^{ \pm}(S) \rightarrow 1 .
$$

We say that a nontrivial element $\gamma \in \pi_{1}(S, b)$ fills $S$ if the curve representing $\gamma$ intersects every essential simple closed curve on $S$.

Theorem 3.2 (Kra's construction Kra81]). Let $S$ be a finite type surface possibly with punctures. Let $\gamma \in \pi_{1}(S, b)$. The mapping class Push $(\gamma) \in \operatorname{Mod}^{ \pm}(S, b)$ is pseudo-Anosov if and only if $\gamma$ fills $S$.

The third ingredient is the following punctured Dehn-Nielsen-Baer theorem; e.g. see e.g. Farb-Margalit FM12, Theorem 8.8]. For a group $G$, denote by $\operatorname{Out}(G)$ the outer automorphism group of $G$; i.e. $\operatorname{Out}(G)=$ $\operatorname{Aut}(G) / \operatorname{Inn}(G)$, where $\operatorname{Aut}(G)$ denotes the automorphism group of $G$ and $\operatorname{Inn}(G)$ denotes the group subgroup of $\operatorname{Aut}(G)$ consisting of conjugations.

Theorem 3.3 (Punctured Dehn-Nielsen-Baer Theorem). Let $S$ be a finite type surface possibly with punctures. Let Out* $\left(\pi_{1}(S)\right)$ (resp. Aut* $\left.\left(_{1}(S)\right)\right)$ be the subgroup of $\operatorname{Out}\left(\pi_{1}(S)\right)$ (resp. Aut $\left(\pi_{1}(S)\right)$ ) consisting of elements that leave invariant the set of conjugacy classes in $\pi_{1}(S)$ of simple closed curves surrounding individual punctures. Then the natural maps

$$
\operatorname{Mod}^{ \pm}(S) \rightarrow \operatorname{Out}^{*}\left(\pi_{1}(S)\right) \text { and } \operatorname{Mod}^{ \pm}(S, b) \rightarrow \operatorname{Aut}^{*}\left(\pi_{1}(S)\right)
$$

are isomorphisms.

Given $f \in \operatorname{Mod}^{ \pm}\left(S_{g, p+n}\right)$, there is an induced action $f_{*}$ on the fundamental group $\pi_{1}\left(S_{g, p+n}\right)$ up to conjugation. Therefore we have an injective homomorphism $\rho: \operatorname{Mod}^{ \pm}\left(S_{g, p+n}\right) \rightarrow \operatorname{Out}\left(\pi_{1}\left(S_{g, p+n}\right)\right)$ given by $\rho(f)=f_{*}$. By post-composing with the embedding of the braid point-pushing subgroup $P B_{n}\left(S_{g, p}\right)<$ $\operatorname{Mod}^{ \pm}\left(S_{g, p+n}\right)$, we obtain an embedding

$$
\theta: P B_{n}\left(S_{g, p}\right)<\operatorname{Mod}^{ \pm}\left(S_{g, p+n}\right) \rightarrow \operatorname{Out}\left(\pi_{1}\left(S_{g, p+n}\right)\right) .
$$

Let $\operatorname{Mod}^{ \pm}\left(S_{g, p, n}\right)$ be the extended mapping class group that fixes two sets of punctures, one with $p$ points and the other with $n$ points. The following proposition computes the normalizer of $P B_{n}\left(S_{g, p}\right)<\operatorname{Out}\left(\pi_{1}\left(S_{g, p+n}\right)\right)$, which is the main ingredient in proving Theorem 1.4. 
Proposition 3.4. For $n>0, g>1$ and $p \geq 0$, the normalizer of $P B_{n}\left(S_{g, p}\right)<\operatorname{Out}\left(\pi_{1}\left(S_{g, p+n}\right)\right)$ is $\operatorname{Mod}^{ \pm}\left(S_{g, p, n}\right)$.

Proof. Let $R \in \operatorname{Out}\left(\pi_{1}\left(S_{g, n+p}\right)\right)$ be an element in the normalizer of $P B_{n}\left(S_{g, p}\right)$. Then $R$ acts as an automorphism $A$ on $P B_{n}\left(S_{g, p}\right)$ by conjugation; i.e. $R \theta(e) R^{-1}=\theta(A(e))$ for $e \in P B_{n}\left(S_{g, p}\right)$. This gives the following equation

$$
R \theta(e)=\theta(A(e)) R \in \operatorname{Out}\left(\pi_{1}\left(S_{g, p+n}\right)\right) .
$$

We claim that for any curve $c$ surrounding a puncture, $R(c)$ is also a curve surrounding a puncture. This implies that $R \in \operatorname{Mod}^{ \pm}\left(S_{g, p+n}\right)$ by Theorem 3.3 .

Let $c$ be a simple closed curve surrounding a puncture. Since $P B_{n}\left(S_{g, p}\right)$ fixes all punctures, we have $\theta(e)(c)=c$ and $\theta(A(e))(c)=c$. By equation (5), we have that $\theta(A(e))(R(c))=R(\theta(e)(c))=R(c)$ for any $e \in P B_{n}\left(S_{g, p}\right)$. However, because of Theorem 3.2, we know that that there is a pseudo-Anosov element $e^{\prime}$ in $P B_{n}\left(S_{g, p}\right)$. Set $e=A^{-1}\left(e^{\prime}\right)$. Then $\theta(A(e))(R(c))=\theta\left(e^{\prime}\right)(R(c))$. Therefore $\theta\left(e^{\prime}\right)(R(c))=R(c)$, which implies that $R(c)$ is peripheral by Theorem 3.1.

What remains to be proven is that $R \in \operatorname{Mod}^{ \pm}\left(S_{g, p, n}\right)$. By the generalized Birman exact sequence (see e.g. Farb-Margalit FM12, Theorem 9.1])

$$
1 \rightarrow P B_{n+p}\left(S_{g}\right) \rightarrow \operatorname{Mod}^{ \pm}\left(S_{g, p+n}\right) \rightarrow \operatorname{Mod}^{ \pm}\left(S_{g}\right) \times \Sigma_{n} \rightarrow 1,
$$

$R$ induces an automorphism $A^{\prime}$ of $P B_{n+p}\left(S_{g}\right)$ by conjugation. We have the following exact sequence given by forgetting the $n$ punctures

$$
1 \rightarrow P B_{n}\left(S_{g, p}\right) \rightarrow P B_{n+p}\left(S_{g}\right) \rightarrow P B_{p}\left(S_{g}\right) \rightarrow 1
$$

Since $A^{\prime}$ preserves the subgroup $P B_{n}\left(S_{g, p}\right) \unlhd P B_{n+p}\left(S_{g}\right)$, it should induce an action on each term of (6). As a result, $R$ fixes the $p$ punctures as a set, which implies that $R$ should also fix the other $n$ punctures as well. Thus $R \in \operatorname{Mod}^{ \pm}\left(S_{g, p, n}\right)$.

Let $\operatorname{Mod}^{ \pm}\left(S_{g, p, n-1,1}\right)$ be the extended mapping class group that fixes three sets of punctures, one with $p$ points, one with $n-1$ points and the last one with one point. Let $\phi: \operatorname{Mod}^{ \pm}\left(S_{g, p+n-1,1}\right) \rightarrow \operatorname{Aut}\left(\pi_{1}\left(S_{g, p+n-1}\right)\right)$ be the natural embedding induced by conjugation on the normal subgroup $\pi_{1}\left(S_{g, p+n-1}\right) \unlhd \operatorname{Mod}^{ \pm}\left(S_{g, p+n-1,1}\right)$. By a similar argument, we obtain the following:

Proposition 3.5. For $g>1, n>1, p \geq 0$, the normalizer of

$$
P B_{n}\left(S_{g, p}\right)<\operatorname{Mod}^{ \pm}\left(S_{g, p+n-1,1}\right) \stackrel{\phi}{\rightarrow} \operatorname{Aut}\left(\pi_{1}\left(S_{g, p+n-1}\right)\right) \text { is } \operatorname{Mod}^{ \pm}\left(S_{g, p, n-1,1}\right) .
$$

We are now ready to prove Theorem 1.4 that is $\operatorname{Mod}^{ \pm}\left(S_{g, p, n}\right) \cong \operatorname{Aut}\left(P B_{n}\left(S_{g, p}\right)\right)$.

Proof of Theorem 1.4. First of all, from another version of the generalized Birman exact sequence (see e.g. Farb-Margalit FM12, Theorem 9.1])

$$
1 \rightarrow P B_{n}\left(S_{g, p}\right) \rightarrow \operatorname{Mod}^{ \pm}\left(S_{g, p, n}\right) \rightarrow \operatorname{Mod}^{ \pm}\left(S_{g, p}\right) \times \Sigma_{n} \rightarrow 1
$$

there is a map

$$
C: \operatorname{Mod}^{ \pm}\left(S_{g, p, n}\right) \rightarrow \operatorname{Aut}\left(P B_{n}\left(S_{g, p}\right)\right)
$$


given by conjugating the subgroup $P B_{n}\left(S_{g, p}\right)$. Let $\left(b_{1}, \ldots, b_{n}\right) \in \operatorname{PConf}_{n}\left(S_{g, p}\right)$ be a base point. The mapping class group $\operatorname{Mod}^{ \pm}\left(S_{g, n, p}\right)$ fixes $\left\{b_{1}, \ldots, b_{n}\right\}$ as a set. Let $T: \operatorname{Mod}^{ \pm}\left(S_{g, p, n}\right) \rightarrow \Sigma_{n}$ be the homomorphism recording to the permutation of the $n$ points $\left\{b_{1}, \ldots, b_{n}\right\}$. Let $f \in \operatorname{Mod}^{ \pm}\left(S_{g, n, p}\right)$. A geometric representation $F \in \operatorname{Diff}^{ \pm}\left(S_{g, p, n}\right)$ of $f$ induces a map $f_{n}: \operatorname{PConf}_{n}\left(S_{g, p}\right) \rightarrow \operatorname{PConf}_{n}\left(S_{g, p}\right)$ by acting on coordinates. Let $\sigma(f): \operatorname{PConf}_{n}\left(S_{g, p}\right) \rightarrow \operatorname{PConf}_{n}\left(S_{g, p}\right)$ be the permutation of coordinates according to $T(f)$. We have that the permutation $\sigma(f)^{-1} \circ f_{n}$ fixes the base point $\left(b_{1}, \ldots, b_{n}\right)$, which induces a map on the fundamental group $\left(\sigma(f)^{-1} \circ f_{n}\right)_{*}: P B_{n}\left(S_{g, p}\right) \rightarrow P B_{n}\left(S_{g, p}\right)$. Geometrically, we have the identification $C(f)=\left(\sigma(f)^{-1} \circ f_{n}\right)_{*}$.

Let $F_{i}: P B_{n}\left(S_{g, p}\right) \rightarrow P B_{n-1}\left(S_{g, p}\right)$ be the forgetful map forgetting the $i$ th coordinate. By Theorem 1.1. given any automorphism $A: P B_{n}\left(S_{g, p}\right) \rightarrow P B_{n}\left(S_{g, p}\right)$, we have that $F_{i} \circ A$ factors through $F_{j}$ for some $j \in\{1, \ldots, n\}$. Therefore we obtain a homomorphism $S: \operatorname{Aut}\left(P B_{n}\left(S_{g}\right)\right) \rightarrow \Sigma_{n}$, where for $A \in \operatorname{Aut}\left(P B_{n}\left(S_{g}\right)\right)$, the image $S(A)$ satisfies that $F_{i} \circ A$ factors through $F_{S(A)(i)}$. Since $C(f)=\left(\sigma(f)^{-1} \circ f_{n}\right)_{*}$ and $\sigma(f)^{-1} \circ f_{n}$ permutes coordinates of $\operatorname{PConf}_{n}\left(S_{g, p}\right)$ by $T(f)^{-1}$, we know that $T(f)^{-1}=S(C(f))$.

By the five lemma, to prove that $C$ is an isomorphism, we only need to show that $C^{\prime}: \operatorname{Ker}(T) \rightarrow$ $\operatorname{Ker}(S)$ is isomorphism. Denote by $\operatorname{Aut}^{0}\left(P B_{n}\left(S_{g}\right)\right)$ the subgroup of $\operatorname{Aut}\left(P B_{n}\left(S_{g}\right)\right)$ consisting of all $A \in$ $\operatorname{Aut}\left(P B_{n}\left(S_{g, p}\right)\right)$ such that $F_{n} \circ A$ factors through $F_{n}$; i.e. $\operatorname{Aut}^{0}\left(P B_{n}\left(S_{g}\right)\right)=S^{-1}\left(\Sigma_{n-1} \times 1\right)$. We have that $T^{-1}\left(\Sigma_{n-1} \times 1\right)=\operatorname{Mod}^{ \pm}\left(S_{g, p, n-1,1}\right)$. By the five lemma, to show that $C^{\prime}$ is an isomorphism, we only need to show that $C^{\prime \prime}: S^{-1}\left(\Sigma_{n-1} \times 1\right) \rightarrow T^{-1}\left(\Sigma_{n-1} \times 1\right)$ is an isomorphism; i.e. $C^{\prime \prime}: \operatorname{Mod}^{ \pm}\left(S_{g, p, n-1,1}\right) \rightarrow$ $\operatorname{Aut}^{0}\left(P B_{n}\left(S_{g}\right)\right)$ is an isomorphism. The action $A \in \operatorname{Aut}^{0}\left(P B_{n}\left(S_{g}\right)\right)$ factors through $F_{n}$, which induces an action of the following commutative diagram:

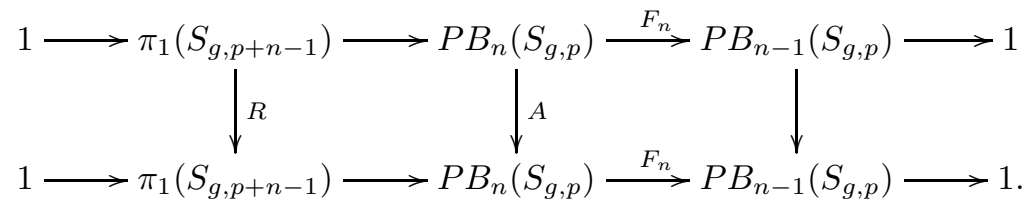

Using this diagram, we can define a homomorphism $P: \operatorname{Aut}^{0}\left(P B_{n}\left(S_{g, p}\right)\right) \rightarrow \operatorname{Aut}\left(\pi_{1}\left(S_{g, p+n-1}\right)\right)$ via the formula $P(A)=R$. We will prove that $C^{\prime \prime}$ is an isomorphism by showing that $P$ is the inverse of $C^{\prime \prime}$. This will conclude the proof of Theorem 1.4

- Step 1: $P$ is injective.

Consider $A \in \operatorname{Ker}(P)$. Therefore $R=P(A)$ is the identity. For any $e \in P B_{n}\left(S_{g, p}\right)$ and $x \in$ $\pi_{1}\left(S_{g, p+n-1}\right)$, we have that

$$
e x e^{-1} \stackrel{R=i d}{=} R\left(e x e^{-1}\right) \stackrel{R=A}{=} A(e) R(x) A(e)^{-1} \stackrel{R=i d}{=} A(e) x A(e)^{-1}
$$

So $e^{-1} A(e) \in P B_{n}\left(S_{g, p}\right)$ commutes with $x \in \pi_{1}\left(S_{g, p+n-1}\right)$. Since the conjugation action of $P B_{n}\left(S_{g, p}\right)$ on $\pi_{1}\left(S_{g, p+n-1}\right)$ is faithful, this implies that $e^{-1} A(e)=i d$. So $A(e)=e$, as desired.

- Step 2: Image $(P)<\phi\left(\operatorname{Mod}^{ \pm}\left(S_{g, p, n-1,1}\right)\right)$.

Consider $A \in \operatorname{Aut}^{0}\left(P B_{n}\left(S_{g, p}\right)\right)$ and set $R=P(A)$. For $e \in P B_{n}\left(S_{g, p}\right)$, denote by $\sigma_{e}$ the automorphism of $\pi_{1}\left(S_{g, p+n-1}\right)$ induced by $e$ via conjugation. For any $e \in P B_{n}\left(S_{g, p}\right)$ and $x \in \pi_{1}\left(S_{g, p+n-1}\right)$, we have

$$
R\left(e x e^{-1}\right)=A(e) R(x) A(e)^{-1} .
$$

That is to say

$$
R \sigma_{e} R^{-1}=\sigma_{A(e)} \in \operatorname{Aut}\left(\pi_{1}\left(S_{g, p+n-1}\right)\right) .
$$

By Proposition 3.5, we have that $R \in \operatorname{Mod}^{ \pm}\left(S_{g, p, n-1,1}\right)$. 
- Step 3: Both $\pi_{1}\left(S_{g, p+n-1}\right)$ and $P B_{n}\left(S_{g, p}\right)$ are normal subgroups of $\operatorname{Mod}^{ \pm}\left(S_{g, p+n-1,1}\right)$. For $f \in$ $\operatorname{Mod}^{ \pm}\left(S_{g, p, n-1,1}\right)$, the image $\phi(f)$ is the conjugation action of $f$ on $\pi_{1}\left(S_{g, p+n-1}\right)$. The image $A=C^{\prime \prime}(f)$ is the conjugation action of $f$ on $P B_{n}\left(S_{g, p}\right)$. The image $P \circ C^{\prime \prime}(f) \in \operatorname{Aut}^{0}\left(\pi_{1}\left(S_{g, p+n-1}\right)\right)$ is the restriction of $C^{\prime \prime}(f)$ on $\pi_{1}\left(S_{g, p+n-1}\right)$, which is equal to the direct conjugation $\phi(f)$. Therefore we have that $\phi=P \circ C^{\prime \prime}$. Therefore the composition $P \circ C^{\prime \prime}: \operatorname{Mod}^{ \pm}\left(S_{g, p, n-1,1}\right) \rightarrow \operatorname{Aut}^{0}\left(\pi_{1}\left(S_{g, p+n-1}\right)\right)$ is an isomorphism onto the image $\phi\left(\operatorname{Mod}^{ \pm}\left(S_{g, p, n-1,1}\right)\right)$. By step 2, this means that $P$ is surjective onto $\phi\left(\operatorname{Mod}^{ \pm}\left(S_{g, p, n-1,1}\right)\right)$. By step 1 , we know that $P$ is also injective. So $P$ is an isomorphism, as desired. This implies that $C^{\prime \prime}$ is an isomorphism as well.

The result for $B_{n}(S)$ follows from a theorem of Ivanov [Iva90, Theorem 2] saying that $P B_{n}(S)$ is a characteristic subgroup of $B_{n}(S)$.

\section{References}

[An16] B. An. Automorphisms of braid groups on orientable surfaces. J. Knot Theory Ramifications, 25(5):1650022, 32, 2016.

[Art47] E. Artin. Braids and permutations. Annals of Mathematics, pages 643-649, 1947.

[Bau63] G. Baumslag. Automorphism groups of residually finite groups. J. London Math. Soc., 38:117-118, 1963.

[Che16] L. Chen. The universal $n$-pointed surface bundle only has $n$ sections. Pre-print: https://arxiv.org/abs/1611.04624, to appear in Jour. of Topology and Analysis, 2016.

[FM12] B. Farb and D. Margalit. A primer on mapping class groups, volume 49 of Princeton Mathematical Series. Princeton University Press, Princeton, NJ, 2012.

[Hem72] J. Hempel. Residual finiteness of surface groups. Proc. Amer. Math. Soc., 32:323, 1972.

[HT85] M. Handel and W. Thurston. New proofs of some results of nielsen. Advances in Mathematics, 56(2):173-191, 1985.

[IIM03] E. Irmak, N. Ivanov, and J. McCarthy. Automorphisms of surface braid groups. Pre-print: https://arxiv.org/abs/math/0306069, 2003.

[Iva90] N. Ivanov. Permutation representations of braid groups of surfaces. Matematicheskii Sbornik, 181(11):1464-1474, 1990.

[Joh99] F. E. A. Johnson. A rigidity theorem for group extensions. Arch. Math. (Basel), 73(2):81-89, 1999.

[Kra81] I. Kra. On the Nielsen-Thurston-Bers type of some self-maps of Riemann surfaces. Acta Math., 146(3-4):231-270, 1981.

[KY11] Y. Kida and S. Yamagata. Commensurators of surface braid groups. J. Math. Soc. Japan, 63(4):1391-1435, 2011.

[KY13] Y. Kida and S. Yamagata. The co-Hopfian property of surface braid groups. J. Knot Theory Ramifications, 22(10):1350055, 46, 2013. 
[Lin04] V. Lin. Braids and permutations. Pre-print: https://arxiv.org/abs/math/0404528, 2004.

[MS74] J. Milnor and J. Stasheff. Characteristic classes. Princeton University Press, Princeton, N. J.; University of Tokyo Press, Tokyo, 1974. Annals of Mathematics Studies, No. 76.

[Sal15] N. Salter. Cup products, the Johnson homomorphism and surface bundles over surfaces with multiple fiberings. Algebr. Geom. Topol., 15(6):3613-3652, 2015.

[Tot96] B. Totaro. Configuration spaces of algebraic varieties. Topology, 35(4):1057-1067, 1996. 